\title{
Is Awareness of Infant Hearing Screening in India Still in its Infancy? A Survey
}

\author{
Archana Rai ${ }^{1 *}$, Reethu Mariam Stephen ${ }^{2}$ and Vibha Vinod ${ }^{2}$ \\ ${ }^{1}$ Lecturer/ASLP, Nitte Institute of Speech and Hearing, Mangalore, India \\ ${ }^{2} B A S L P$, Nitte Institute of Speech and Hearing, Mangalore, India
}

Submission: March 14, 2019; Published: March 26, 2019

*Corresponding author: Archana Rai S, Lecturer, Nitte Institute of Speech and Hearing, Medical Sciences Complex, Mangalore, India

\begin{abstract}
The origin of newborn hearing screening dates back to the 1990s. Since its inception, newborn hearing screening has helped developed countries in early identification and early intervention of hearing loss. However, in a developing country, like India, newborn hearing screening is performed in a few hospitals and private setups, on the basis of reference given by the pediatricians. Regrettably, recommendation for hearing screening is not provided to all the infants. To ensure all infants undergo newborn hearing screening there must be increased awareness regarding the importance of hearing screening among the public. This is a study conducted to check the public's awareness of newborn hearing screening in India. A self-made questionnaire was designed and circulated in a time-bound manner to the public via Google form platform. 350 respondents' data were collected and tabulated for further analysis. The result of the study shows, a lack of awareness of newborn hearing screening in the Indian population. The outcome of the study is, a serious need for spreading awareness regarding hearing loss in infants and the importance of hearing screening.
\end{abstract}

Keywords: Newborn; Hearing Screening; Hearing Impairment; Awareness

\section{Introduction}

Hearing impairment (HI) is one of the most prevalent sensory deficits in India; out of 1000 neonates approximately 5-6 infants are diagnosed with HI [1]. According to the Rehabilitation Council of India (2000) [2], 4 out of every 1000 live birth were found to have severe to profound hearing loss. HI can occur as early as at birth, and is termed as congenital hearing impairment, usually goes unnoticed by parents and by caregivers. $\mathrm{HI}$ is an invisible handicap, leading to impaired social communication, language development and quality of life. A neonate born with hearing impairment appears as healthy as any other normal child. But the $\mathrm{HI}$, if it is overlooked during the early stages of life, might have an adverse effect on the central auditory pathway development which in turn will inhibit the primary and secondary language development $[3,4]$. Therefore, early detection is a vital element in providing appropriate support for children with hearing impairment which would help them to enjoy equal opportunities in society alongside their peers [5]. Children who receive timely rehabilitation and management are found to have better linguistic skills than those who are not provided with early rehabilitation [3]. Earlier, only those neonates who were in the "high-risk register" were screened, over the years, it was noticed that more than half of the children with hearing impairment were overlooked because they were not high-risk babies.
Therefore, it is advised to screen all the infants regardless of their falling in the high-risk category or not [6]. Amongst all the medical institutions in India only $38.09 \%$ carry out newborn hearing screening, which implies that more than half of the medical setups do not carry out newborn hearing screening [7]. Hence, children who are born in these setups and whose parents are unaware of UNHS are at a higher risk of being missed out. Hearing screening is a rapid assessment tool of audiology that indicates the result as pass or fail. In case the test results show fail, it signifies the probable presence of Hearing Loss (HL), and the child is recommended for a detailed evaluation. On the other hand, if the results show pass, it implies the absence of HI. The concept of Universal Newborn Hearing Screening (UNHS) was advocated in the year 1999 by the American Academy of Paediatrics. They recommended, "Universal hearing screening of all infants" which was then trailed by several other organizations. In 2000, the Joint Committee on Infant Hearing (JCIH) [8] put forth a set of fixed guidelines for how to carry out UNHS programs and to arrive at an early diagnosis, as to provide early intervention and counseling to the family. According to JCIH (2007) [9], the infant should be screened within the first month of birth and if hearing impairment is present, diagnosis and rehabilitation measures should be adopted before 3 months of age. Further, speech and language 
intervention should be initiated by the first 6 months of life for the successful integration of the individual into society and to help them lead a productive life $[9,10]$.

Generally, a UNHS program is supposed to differentiate between normal-hearing infants and those with HI, diagnose the presence of $\mathrm{HI}$ as early as possible and provide adequate management options [11-13]. In addition, an effective UNHS program will aim to lessen and/or prevent HI which may be a risk for the normal linguistic, emotional, social and cognitive development in neonates [13]. India being a developing country, awareness of certain issues is still lacking. One of those is the early identification of hearing loss. To our knowledge, newborn hearing screening (NHS) is not practiced in all audiological setups/ENT/hospitals. Moreover, awareness of NHS in public is not documented till date. The present study aims to understand the level of awareness about newborn hearing screening in the public. The objective of the present study also helps to identify the level of awareness in males and females and also between medical and non-medical professionals.

\section{Method}

A questionnaire was formed with questions including the general demographic data and more specific questions aimed at gauging the knowledge of an individual about UNHS. Total of 23 questions were formulated including both subjective and objective type. This was given for validation to 11 professional Audiologist and Speech Language Pathologists. The corrections and suggestions were incorporated into the questionnaire. Once the questions were finalized, it was circulated to the common public via Google forms. The responses obtained were transferred to an excel sheet where the data analysis was carried out. 50 responses were eliminated as the respondents were professional who perform the NHS. Further descriptive statistical analysis was done. The results are tabulated in the form of a pie chart.

\section{Results}

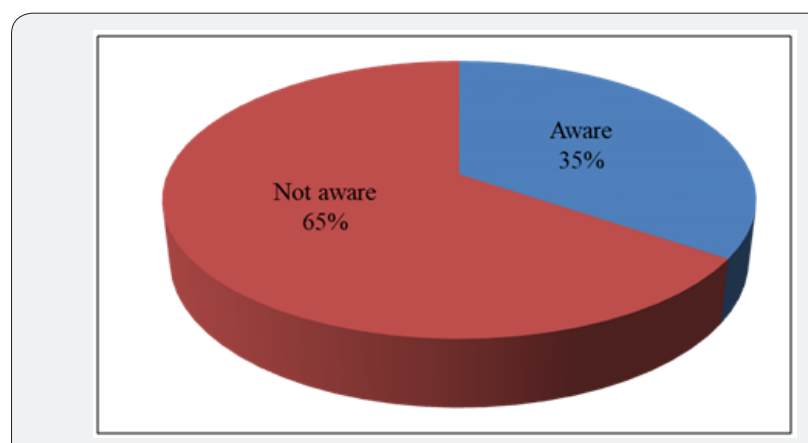

Graph 1: Awareness of UNHS among the population tested.

The current study focused on exploring the public's awareness of UNHS from different parts of the country. Total of 400 responses was recorded and out of these 50 responses were eliminated since the responses were from Audiologists and Speech-Language Pathologists. Results of descriptive statistical analysis are depict- ed below. (Graph 1) represents awareness of UNHS. Among the 350 participants, merely 121 (35\%) respondents were 'aware of UNHS' and 229 (65\%) respondents were 'not aware of UNHS.' (Graph 2) depicts awareness of UNHS in males and females. The study noted $52 \%$ of the total respondents were females and $48 \%$ were males. Of the $52 \%$ female respondents, $21 \%$ of them reported being 'aware of UNHS' and $31 \%$ as 'not aware of UNHS'. On the other hand, out of the $48 \%$ of male respondents, only $14 \%$ of the respondents reported 'aware of UNHS' and 34\% reported 'unaware of UNHS'. Overall, the results reveal female respondents were more aware of UNHS compared to UNHS.

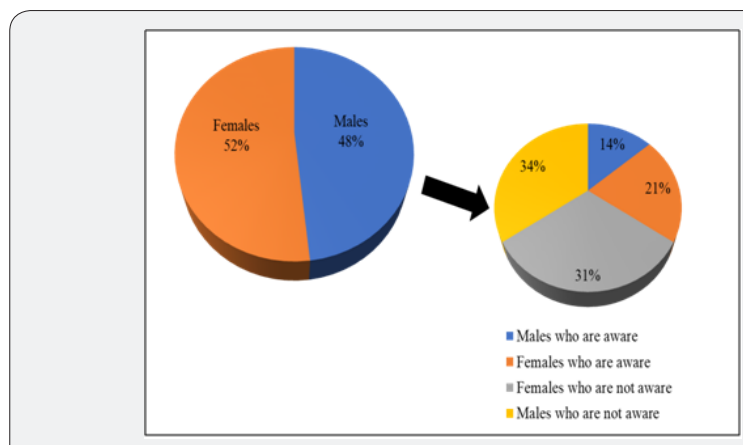

Graph 2: Awareness of UNHS across gender.

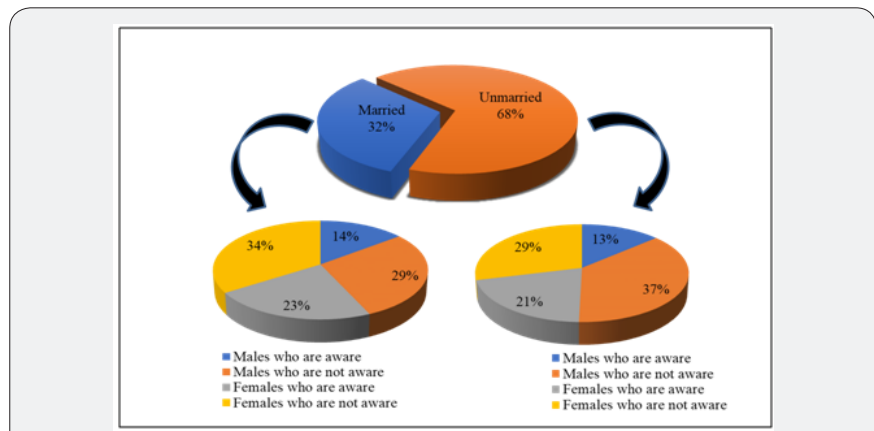

Graph 3: Awareness of UNHS in married and unmarried population.

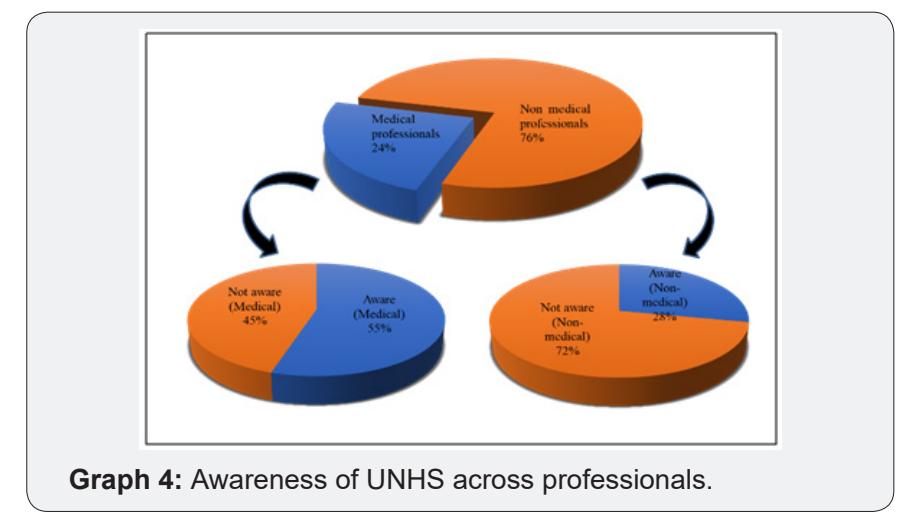

As represented in (Graph 3), 68\% of the respondents were unmarried and $32 \%$ were married. In both groups, female respondents were more aware of UNHS in comparison to males. (Graph 4) indicates the level of awareness among medical and non-medical professionals. Out of the total population of the respondents, 


\section{Global Journal of Otolaryngology}

$24 \%$ were from medical profession (MBBS, dental and paramedical) and $76 \%$ from the non-medical profession (engineering sector, sales/marketing sector, or any other). Awareness of UNHS was found to be more among medical professionals (55\%) when compared to non-medical professionals (28\%). (Graph 5) represents the awareness among individuals belonging to nuclear families versus joint families. Individuals who reside in the Nuclear family were more aware compared to the joint family; however, the difference was not clinically significant (35\% and 33\%) respectively. (Graph 6) represents responses of awareness of hearing loss to be congenital in origin amongst the married individuals. The study had 350 respondents, but only $32 \%$ of the groups belonged to the married category. In this $32 \%$, female respondents were maximally aware of the congenital origin of hearing loss.

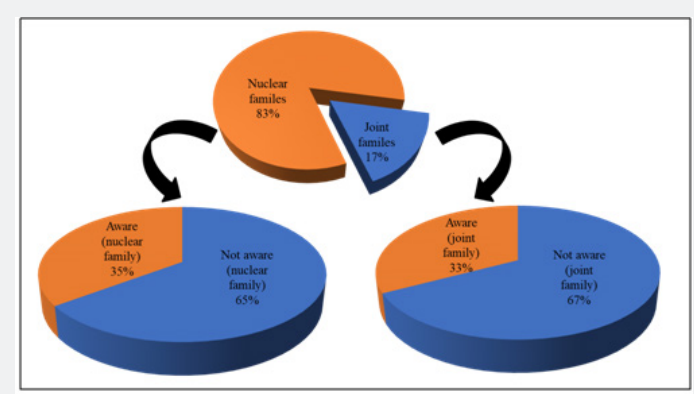

Graph 5: Awareness of UNHS amongst types of families.
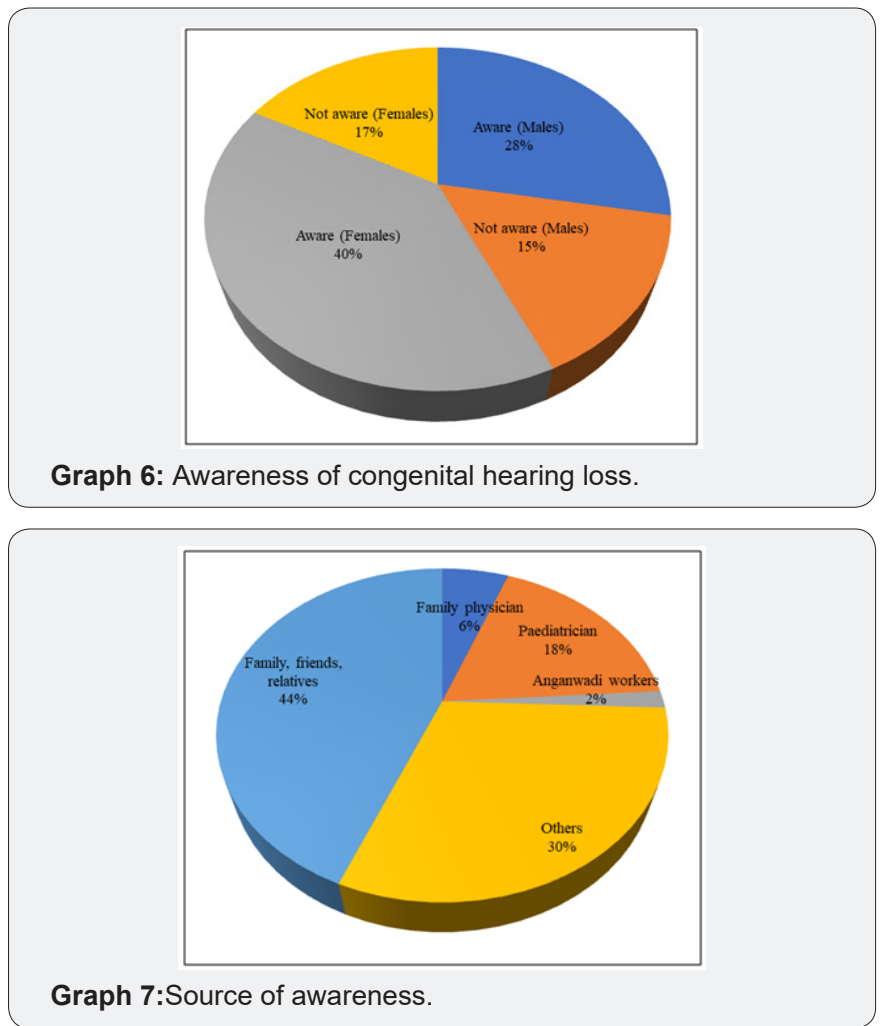

Graph 7 depicts source/means through which the respondents were aware of NHS. Friends, family, and relatives were the source of awareness as reported by $44 \%$ and this source was highest in the hierarchy. Remaining sources of awareness on
UNHS were by others (33\%), pediatrician (18\%), family physician (6\%). The least awareness was found to be spread by Anganwadi workers (2\%). (Graph 8) representing the knowledge about who does UNHS among the people who are aware of newborn hearing screening. $69 \%$ of the respondents were very well aware that audiologists are the ones who does NHS. (Graph 9) depicting the total participants among those who were aware of UNHS; a majority (76\%) of respondents knew what exactly UNHS is out of the options given, i.e., a protocol for hearing assessment in children. However, $16 \%$ of them selected that it is a vaccination for children, $5 \%$ of them selected the option as others. A very small minority of them selected $2 \%$ and $1 \%$ as a disorder in children and not sure respectively. The (Graph 10) indicates the responses recorded for where is UNHS done. Around $72 \%$ of the respondents had opted for the appropriate answer, i.e., Hospital /clinic, 13\% of the respondents selected the option as not sure and $7 \%$ as not aware. However, $4 \%$ of them selected Anganwadi, $2 \%$ as schools and the remaining $2 \%$ as home respectively.
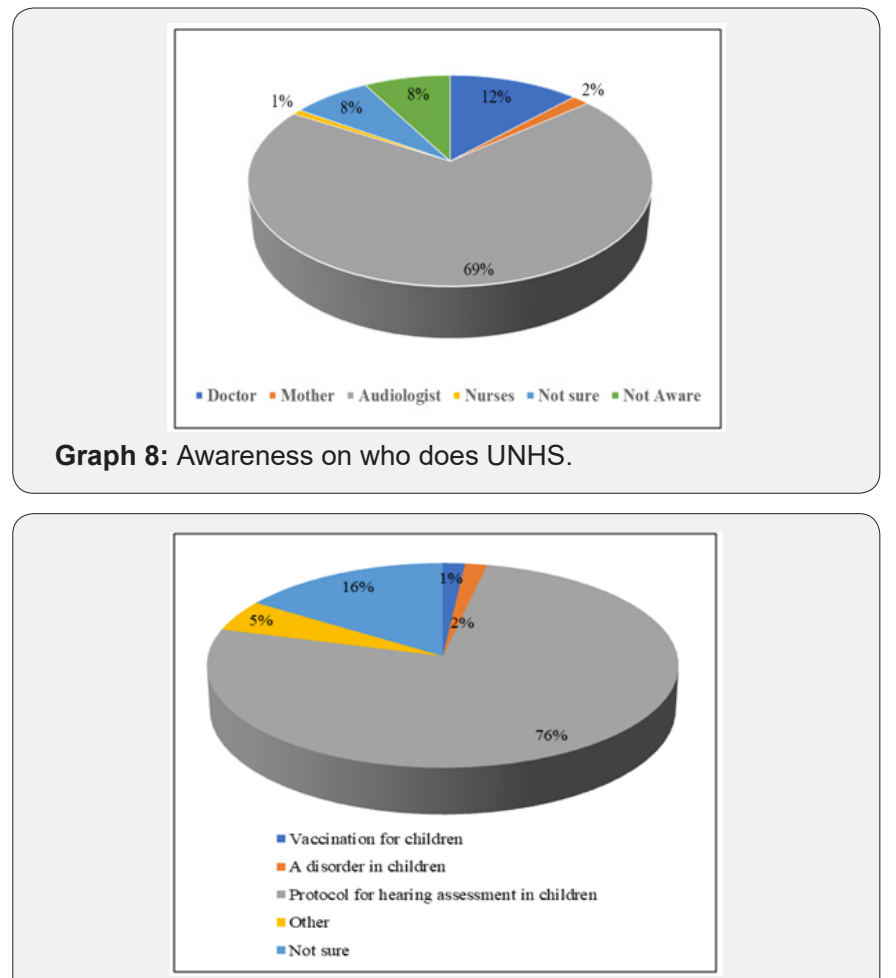

Graph 9: Awareness of what is UNHS

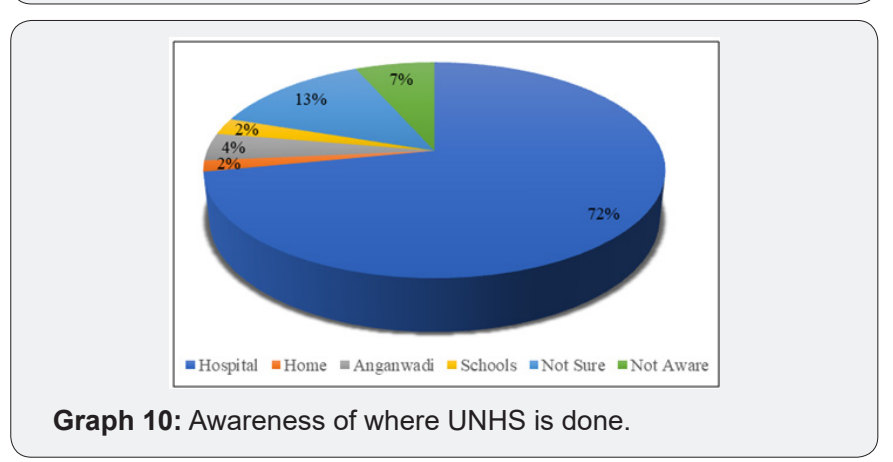




\section{Discussion}

The present study aimed at identifying the awareness of NHS among the public in the developing country like India. Results revealed that only $34.57 \%$ are aware of UNHS. This dearth could be due to a variety of factors like reduced screening coverage, a smaller number of awareness programs or advertisements, etc. It could also be attributed to the fact that UNHS is still in its infancy and is not being mandatorily practiced throughout the country. Results revealed that females when compared to males, had an upper hand in awareness regarding newborn hearing screening. This could be because neonates who undergo various tests in the early days of their lives are usually accompanied by mothers or by the females of the family. Hence, they are more exposed to the tests which are undergone by the neonates and are likely to have better knowledge in this area than males. Additionally, the female members in the family aid the mother in the postnatal care of the baby. Thus, increasing the probability of being aware of newborn hearing screening. Individuals, who were married, comparatively are more aware of UNHS. This could be due to the information acquired via various counseling programs for married couples regarding family planning and parenting. Parents, in particular mothers, seem to be more aware of matters related to the hearing loss in infants as they tend to be more concerned and thoughtful towards the needs and requirements of children and issues related to them.

Medical professionals are more exposed to scenarios of childbirth and childcare and possess academic knowledge, and thus are significantly more aware of UNHS. Also, they are in part responsible for counseling the expectant and new mothers about hearing screening and the consequences of not undergoing NHS. Further, as medical professionals work in a team setup, they are more aware of the roles and duties fulfilled by other medical and paramedical lines. Thus, medical professionals being more aware of UNHS can be attributed to the aforementioned factors. Even so, as nearly half of the respondents from the medical professions are unaware of the hearing screening program there are high chances that a big amount of infants may go unnoticed and unscreened. Educating the medical professionals about newborn hearing screening can go a long way in bringing those neonates who are missed out under the radar. Even the people who seem to have heard of UNHS are not aware of what exactly the program does. About one-fourth of the respondents who had heard of UNHS responded incorrectly to the question of what is UNHS or who does UNHS.

This could be due to the fact that even though there is a nominal awareness of the field among the public, they are not informed of the duties carried out by the Audiologist and Speech Language Pathologist. However, the growing popularity of the field of audiology and speech-language pathology can be a contributing factor to the awareness of the majority of the individuals regarding the professionals involved in carrying out the newborn hearing screening. In general, this lack of awareness of UNHS can be re- garded as an eye opener for all the professional ASLPs to be more proactive and adopt effective awareness strategies by means of counseling, organizing awareness camps and exhibitions, distribution of pamphlets/ posters, conducting workshops, etc. The limitations of the present study are, the questionnaire was circulated mainly through social media, so only the literate and those who are digitally accessible were able to participate. As the questionnaire was objective type, there are chances of the answers being guessed which could not be controlled.

\section{Conclusion}

The present study aimed at identifying the level of awareness regarding UNHS among the public population. It was found that there is an overall lack of awareness of UNHS in the Indian population. Hence, there is a pressing need for spreading awareness regarding hearing loss in infants and the importance of hearing screening. The Govt. of India as well as private health clinics should undertake measures for the same as a part of its public health plans. Also, the professionals working in the field of audiology should take initiatives to spread awareness by the means of awareness camps, workshops, seminars and street play activities in different work sectors, public gathering and through mass media. With respect to rehabilitation programs, screening measures should be brought under the kaleidoscope as early detection and management are vital in preventing or mitigating the negative effects of disability. The present study can also contribute to the enrichment of literature in the field of audiology.

\section{References}

1. Garg S, Singh R, Khurana D (2015) Infant hearing screening in India: current status and way forward. International journal of preventive medicine 6 .

2. Kundu CL (2000) Status of Disability in India 2000 New Delhi: Rehabilitation Council of India, Ministry of Welfare, Government of India.

3. Low WK, Pang KY, Ho LY, Lim SB, Joseph R (2005) Universal newborn hearing screening in Singapore: the need, implementation, and challenges. Ann Acad Med Singapore 34(4): 301-306.

4. Koop CE (1993) We can identify children with hearing impairment before their first birthday. seminars in Hearing 14(1): 1-2.

5. World Health Organization (2010).

6. Sukumaran TU (2011) Newborn hearing screening program. Indian pediatrics 48(5): 351 .

7. Kumar S, Mohapatra B (2011) Status of a newborn hearing screening program in India. International journal of pediatric otorhinolaryngology 75(1): 20-26.

8. Evelyn C (2000) Year 2000 position statement: principles and guidelines for early hearing detection and intervention programs. American Journal of Audiology 9(1): 9-29.

9. Joint Committee on Infant Hearing (2007) Year 2007 position statement: principles and guidelines for early hearing detection and intervention programs. Pediatrics 120(4): 898-921.

10. Winston-Gerson R, Ditty KM (2018) Newborn Hearing Screening. In: National Center for Hearing Assessment and Management. A Resource Guide for Hearing Detection and Intervention p. 1-14. 
11. Majid AH, Zakaria MN, Abdullah NA, Hamzah S, Mukari SM (2017) Current development, issues, and challenges of a universal newborn hearing screening program in Malaysia. International educational scientific research journal 3(4).

12. Clemens CJ, Davis SA, Bailey AR (2000) The false-positive in universal newborn hearing screening. Pediatrics 106(1): e7.
13. Kemp AA, Delecrode CR, Silva GC, Martins F, Frizzo AC, et al. (2015) Neonatal hearing screening in a low-risk maternity hospital in São Paulo state. Brazilian journal of otorhinolaryngology 81(5): 505-513.

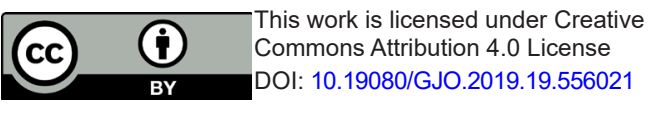

